\title{
Smart Security Solution for Women using IoT
}

\author{
Shivani Dongre, Chetna Amborkar, Ruchi Kotangale, Nikita Kale , Ashiwini Dhoke \\ Prof. Dr. (Mrs.) A. V. Dehankar \\ Department of Computer Technology \\ Priyadarshini College of Engineering, Nagpur, India, 440016
}

\begin{abstract}
Today Government of India have many proposals and facilities for women safety purpose line Android apps, Toll free number, dedicated police team etc. To enhance the safety and security of the women in society we decided to work on the device that is portable and wearable. So many researchers already doing the same but we are motivated to enhance the features and compact device.

IoT is the growing technology day by day and using billions of devices online daily for data storing worldwide. For the project development the IoT plays an important role to get the geometric coordinates on the database as well as body temperature as per the researchers did the research.
\end{abstract}

Keywords- Node MCU ESP, women safety device, IoT

\section{I- LITERATURE REVIEW}

T oday, we are technology growing as digital media and devices are updated day to day. Still women area is the main issue regarding the safety and security. The Factories Act 1948 to allow women employees to work in night shifts so the security is important for them due to the poor thinking men. There are so many researchers who are working for the same and so the review paper to get the idea into reality for internet of things based wearable device that has a trigger button which sends the SMS with the GPS location to the concern person. Internet of things is used to send the location on cloud server database so we can monitor the path on Google map.

Internet of Things term generates from Internet of Everything by Cisco later on the devices is the main part to transmit the data on cloud server then Internet of Things is the name. Things mean the sensors or controllers who are responsible to send the data online.
For women security the Things are GPS and Temperature sensor. The main microcontroller is ESP12 which is 32-bit controller and ability to connect to the internet using programming.

\section{II-LITERATURE REVIEW}

Author described the working of the security assistance device along with its construction. The main components of the device are GSM, GPS, Arduino UNO and ESP8266.The function of GSM is to send the SMS to the present number once the reset button on the arduino UNO which acts as the panic switch of the device is pressed. On receiving the message, with the help of the web server that using NODE MCU which uses google maps, the police control team can track the location of the device and provide help.

GSM is having SIM card to send SMS with GPS location data to the respective mobile number. ESP8266 is the WiFi internet device that sends the same data to the cloud server. The GPS location send via SMS opens the Google map app to see the current location of the device or person.

This paper consists of Smart phone connected to hardware through IOT. The device communicates with smart phone through android app software. The device has the pulse rate $\&$ temperature sensor of the body $\&$ is continuously monitored by the software which is installed in the laptop. The pulse sensor is used to detect the pulse rate beats per minute using arduino programming. The pulse sensor works on the principle of light intensity receiver. The temperature sensor is digital sensor that senses the body temperature. Things peak cloud server is used as iot server that can have the pulse and temperature data in the form of graph. The data is visible online and can be accessible to everyone if it is publically open granted. otherwise login details required to see the data online. 
In cases of abuse, the software perform the tasks like Sends SMS to the family members along with the google map location. Message also sent to the nearest police station requesting immediate action.

The SMS consists of the GPS coordinates longitude and latitude captured by the mobile phone GPS and SMS is sent to the person via SIM card of the mobile phone. In this paper, the goal was to create a wireless button that can trigger a programmed. This project used for: Send instant notifications, SMS messages to relatives. When the button is pushed, receive an alert on mobile phone app. The wireless button device is transmitter RF $433 \mathrm{Mhz}$ device that is connected to encoder and decoder IC at transmitter and receiver module. The transmitter encoder IC is HT12E that send data to RF transmitter and receiver Decoder receive the signal via RF receiver and send a signal to the controller. The transmission is ASK transmission.

This paper the author gives information about the device consists of Smart phone connected to a Smart Band through Bluetooth Low Energy (BLE). The device communicates with smart phone acts as an interface between the device and the phone. It has smart band with sensors such as pulse rate, temperature sensor of the body. The data from the pulse and temperature sensor is sent to the cloud server continuously. The Bluetooth is used to send the data from the device to a mobile phone. The mobile phone has an application to get the data from Bluetooth and send it on the iot using phone gps and internet facility.

In cases of abuse, the app directs the phone to Sends message to the family members along with the coordinates. Also sends information to people in the near vicinity requesting public attention. The app uses the GPS of the smartphone to track the coordinates. The help message is sent to the family members and the nearest police station through the GSM.

In this paper the author uses various sensors are used to analyze the real time situation of a human being like heart beat sensor and some sensors like GPS/GSM are used to identify the location, etc. For the heart pulses the pulse sensor is used that detected the heart beats, the temperature sensor senses the temperature of the body when it is touched on the body, both sensors data pin is connected to arduino microcontroller. The controller then sends the data to IoT server using ESP8266 WiFi device.
Device can identify the situation of a woman in the aspect of women's safety and security alert by gathering all these sensors data with its predefined levels in programming. This research proposes a Low Cost device model that can be affordable to purchase. For future enhancement the surveillance feature may be added to send the image to the police station or friends and family member from men's or intruders.

This paper provides information about the IoT server called Ubidots. The mobile app developers can use these servers to capture the data from the sensors and send it to Ubidots server. The data from the temperature and pulse sensor is sent on the server for the necessary actions and alerts. Realtime GPS locations are accessed by satellite signal sent to the controller and SMS messaging services to be preprogrammed to generate the alerts and it sends to the guardians, relations or nearby police station. By these measures, we could take immediate action. Sample screen of ubidot cloud server platform.

\section{III- HARDWARE REVIEW}

WeMos ESp12: The ESP12 is 32bit controller which have 8 Digital Input output pins and 1 analog pin. $802.11 \mathrm{~b} / \mathrm{g} / \mathrm{n}$, Integrated low power 32bit MCU, Integrated TCP/IP protocol stack, Frequency range : $2.4 \mathrm{GHz}-2.5 \mathrm{GHZ}$, Operating voltage : $3.0 \mathrm{v} \sim 3.6 \mathrm{v}$, Programmable from Arduino Software.

It is developed by AI Thinker labs and it is family of ESP8266. It has $64 \mathrm{kB}$ ROM, 64kB RAM and 96kB data RAM.The silicon chip is housed within a $5 \mathrm{~mm} \mathrm{x}$ $5 \mathrm{~mm}$ package with 33 connection pads, that is 8 pads on each side and one thermal/ground pad at the centre with 9 GPIO pins

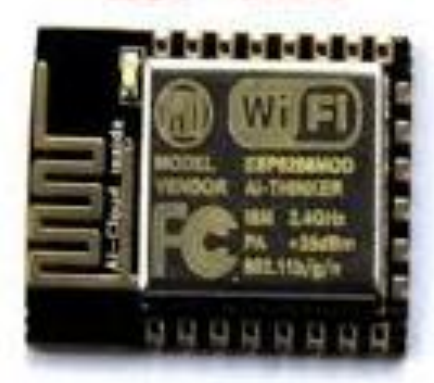

Fig 1. ESP 12E GSM SIM 800L: 
Quad-band 850/900/ 1800/ 1900MHz - connect onto any global GSM network with any 3G/4G SIM Operating Voltage 3.7V Operating Current $800 \mathrm{~mA}$

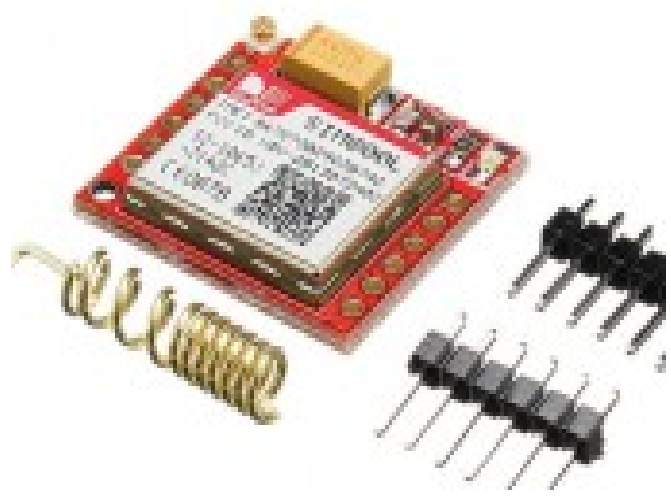

Fig 2 SIM 800L

SIM module is used to send the data using AT commands. The on-board LED starts blinking according when it connects to the mobile network. If the power is not enough it will receive poor signal and it fails to connect to the mobile network. To send or read SMS from the module we need to set the module to SMS mode and then use the AT Commands for SMS.

To set the GSM Module in SMS mode, The AT command is "AT $+\mathrm{CMGF}=1$ ", Once it returns $\mathrm{OK}$ reply then it is set into SMS mode. To send SMS to the mobile number AT+CMGS="9XXXXXXXXX" this command is used. After that the message has to print to send on that number. After sending SMS to exit from the SMS mode "Char 26" has to send. Every time to send the SMS to the respective number the AT commands has to use with the mobile number and message. The SIM module is connected to the transmitter pin of controller to send the AT commands serially.

\section{GPS Module :}

The NEO-6M is a family of stand-alone GPS receiver. 5 Heartz position update rate, E-EPROM for configuration settings, rechargeable $1.5 \mathrm{~V}$ battery for Backup. On board real time clock. Easily connect to GPS satellite and gets the longitude and latitude coordinates.

Operating voltage: 3.3 to $5 \mathrm{~V}$, it is Serial Communication device with Microcontroller

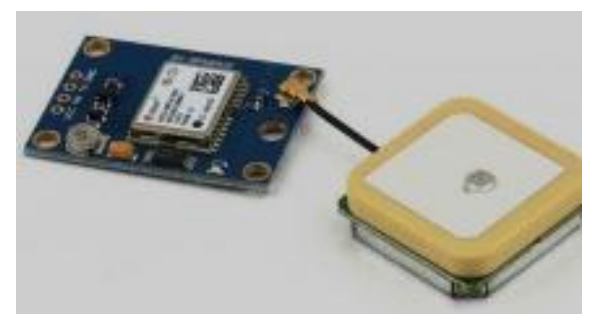

Fig 3. GPS

The GPS is interfaced with the controller by using library called TinyGPS and it detects the coordinates when the GPS is connected to satellite its LED starts blinking and the data is sent to the controller.

This GPS module is used worldwide for research and development purposes. Its drawback is only one that unless it connect to satellite it will not send geo data.

\section{Temperature Sensor :}

DS18B20 it is digital sensor and uses one data pin for communication, it measures temperatures from $-55^{\circ} \mathrm{C}$ to $+125^{\circ} \mathrm{C}$ in $0.5^{\circ} \mathrm{C}$ increments. It requires the library called dallastemperature library in arduino for the programming. Simply by adding the library we can get the data of temperature.

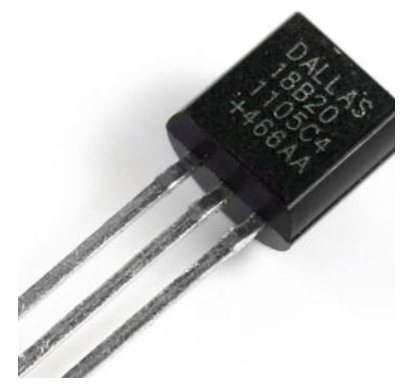

Fig.4 DS18B20

The sensor is directly connected to the digital pin of controller and same pin is connected to Vcc with $4.7 \mathrm{~K}$ resistor.

This sensor is easy to program in arduino as everything is written in its library and we have to use only function call to get the temperature.

\section{IOT Cloud Server:}

The cloud server is the service of domain name and web hosting offered by so many companies online, some of them are ready with API keys like Adafruit or Thingspeak. These servers are able to create the database directly after login into it. They generate the API keys and we have to use this key in program to send the sensor data on the server in our account only. These are called third party servers that has backend 
done already and we have to use API key only. The other way to use the cloud server is to get the hosting service includes MySQL database and supports PHP scripts. We have to create the database in MySQL and then to create the table into it. The columns have to be created in the table.

Now to send the data on that database from the sensors and controller we have to create PHP GET script that will get the sensor data from controller and insert into the database table of the cloud server. This is purely backend PHP program we can use also the front end PHP \& HTML code is used to see the table on the website having MySQL data visible on web page.

The domain name and hosting are paid service provided by various organizations. Free hosting is also available but under the subdomain name provided by 000webhost.com or freehosting.com but having limit of only one database and subdomain, $100 \mathrm{MB}$ space and it will block the IP if we continue to send data on it within 20 second.

\section{IV- PROPOSED METHODOLOGY}

After reviewing the papers by researchers who did the task for the women security and safety, we would like to design the wearable band that consists of the controller ESP12 (IoT enabled) WeMOS D1, GSM and GPS. The ESP is WiFi microcontroller that can be programmed to interface with GPS and GSM. Is is small and so compact as we can wear on the wrist. The GSM is SIM 800L which uses micro SIM card and so it is also compact, GPS is neo gps again very compact. The whole system is powered by lipo battery which is rechargeable and can be recharged with the mobile charger.

For the programming we use Arduino IDE software as it is easy to understand and all the libraries of GPS and GSM is inbuilt in it so programming to wemos is easy way. We have to install ESP library on Arduino IDe and after that it is ready to programming.

The push button is on the device so that the current GPS location is sent to the pre-programmed mobile number using GSM SIM card. The SIM card must have SMS pack enabled.

For cloud server Think speak server is used that has API key and the sensor data will send on thing speak server and it can accessible online from internet.

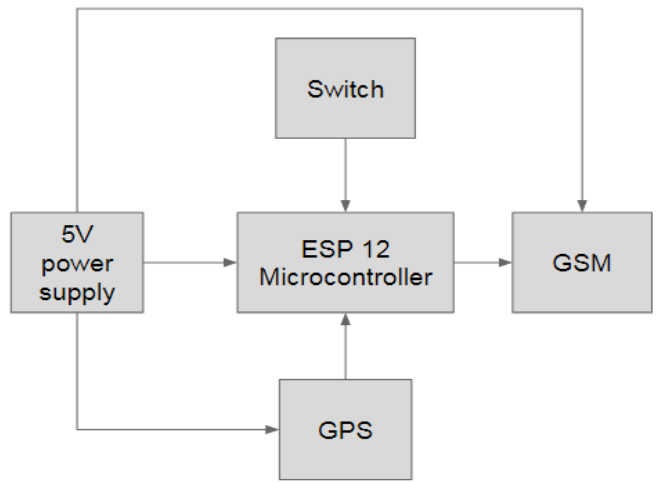

Fig.5. Block Diagram

As per the block diagram the ESP12 controller we will use Wemos as it is tiny and inbuilt programmer connector is provided.

The system is fixed on the wearable belt and with the emergency button on it. If any abuse or emergency occur then button has to be pressed. As soon as button pressed the GPS location data is sent on mobile number that is programmed in controller via GSM module which have SIM card with SMS credit balance.

Also the GPS location data is stored on the cloud server every $5 \mathrm{~min}$ so that we can trace the path on google map.

\section{REFERENCES}

[1] T. Sai Pranay, Y.Megha et al, Security Assistance Device Using Wireless Technology For Women, International Journal of Pure and Applied Mathematics Volume 119 No. 12 2018, 16743-16748[2] Kavya.K , Pavithra.M , Pavithra.N ,IOT Based Women's Safety Gadget, International Journal of Innovative Research in Science, Engineering and Technology An ISO 3297: 2007 Certified Organization Volume 7, Special Issue 1, March 2018

[2] G C Harikiran,Karthik Menasinkai, Smart Security Solution for Women based on Internet Of Things(IOT) , IEEE International Conference on Electrical, Electronics, and Optimization Techniques (ICEEOT) 2016

[3] B.Umadevi, Research Scholar, WOMENS SECURITY SOLUTION USING: IOT, International Journal of Pure and Applied Mathematics Volume 119 No. 10 2018, 1871-1874

[4] Rhythm Kr Das Student of Computer Application Women Safety device (IOT Based)

https:// www.researchgate.net/publication/318381243

[5] WOMENS SECURITY SOLUTION USING: IOT , International Journal of Pure and Applied Mathematics Volume 119 No. 10 2018, 1871-1874. 